\title{
On the structure of the floating zone in melting $\dagger$
}

\author{
I. DA-RIVA AND J. MESEGUER \\ Laboratorıo de Aerodınámıca, E T S I Aeronáutıcos, Unıversıdad Polıtécnıca, Madrıd, Spaın
}

(Recelved 1 January 1978)

\begin{abstract}
Floating zone melting is used in crystal growth and purification of high melting materiass The use of a reduced gravity environment will remove the constraint imposed on the length of the zone by the hydrostatic pressure The equilibrium of the floating zone may involve, (1) Hydrostatic forces, when the zone rotates as a whole (2) Convective driving forces, when the rone is stationary but fluid property gradients appear (3) Hydrodynamic forces, when some parts of the zone dre set into motion with respect to others The last effects are considered in this paper I he flow pattern of a floating zone held between two discs in relative motion is complicated, and thence the solution of the problem is difficult even assuming a constant property-newtonian tiquid Nevertheless, when a small parameter appears in the problem, the complete flow field can be splut into rones where simple solutions are found To illustrate this approach, the spin up from rest of an initally cylindrical floaling zone is considered with detall Here the small parameter is the time elapsed from the impulsive starting of motion Since the problem which has been considered, as well as some others which can be tackled by use of similar methods, concern the viscous layer close to etther plate, they can be simulated experimentally in the ground laboratory with short floating zones Procedures to produce these zones are indicated
\end{abstract}

\section{Introduction}

Floating zone melting has been widely used for the last years in crystal growth and in purification of high melting materials As it can be seen in Fig 1, the melt does not require any crucible since it is held by surface tension forces

In the ground laboratory, the maximum stable length of a vertically suspended liquid zone is controlled by the balance between hydrostatic pressure, which increases with the distance to the top of the molten zone, and surface tension forces. The use of a reduced gravity environment removes the constraint imposed on the length of the floating zone, rendering it more inherently accessible than on earth.

The stability of equilibrium of the floating zone under the large variety of disturbances that could arise either accidentally or intentionally in the space laboratory is a matter of great concern Its study involves a formidable task both because of the material characteristics of the melt, whose properties are strongly temperature dependent, and because of the complexities associated to the disturbances which could be imposed on the zone. Thence, several simplifications must be introduced in the model. The simplest approach consists in disregarding phase changes, considering a liquid zone held between two parallel, coaxial, solid discs.

†Paper presented at the XVIIIth International Astronautical Congress of the International Astronautıcal Federation, Praha, Czechoslovakıa, 25 September-1 October 1977 

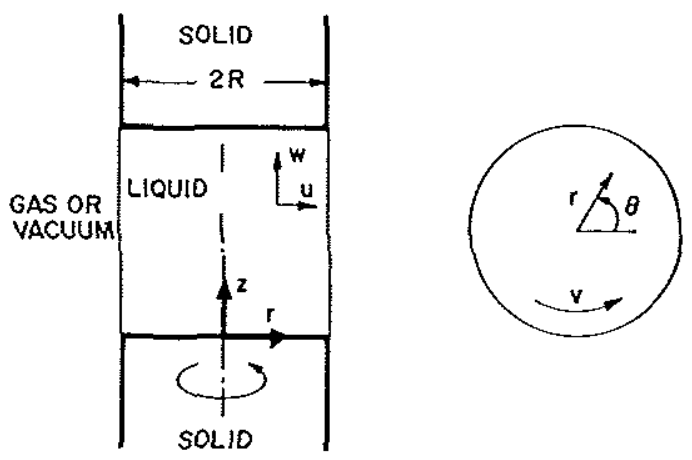

Fig 1. Geometry, coordinate system and fluid velocity components for spinning floating zone.

Among the disturbances that one can impose on a floating zone (heating, vibration, electric or magnetic fields, ...) the most simple one is rotation around the symmetry axis. The aim of this rotation is to homogenize the temperature field.

Studies on the stability of the floating zone could be classed in either of the following categories:

1. Hydrostatic studies. $\dagger$ The zone rotates as a whole. Both the properties of the liquid constituting the zone and the angular velocity of the fluid around the symmetry axis are assumed to be uniform.

2. Studies on naturally induced convection (Chang and Wilcox, 1976; Chun and Wuest, 1977). Now the zone is held at rest, but material property gradients appear as a consequence of heating, intentional diffusion of species, contamination,.... These gradients in the presence of body or surface forces induce the convection.

3. Studies on artificially induced convection (Carruthers and Grasso, 1972; Carruthers et al. 1975). These studies are concerned with the response of the column to a directly applied force which gives rise to a more or less complicated fluid pattern. This is the case when one of the end discs is set into motion.

In this paper we discuss some problems concerning the rotating motion of an incompressible, newtonian, viscous liquid, having constant material properties, which is held by surface tension forces between two parallel coaxial discs.

The mathematical formulation of the problem is moderately complicated, but the solution of the equations involved presents insurmountable difficulties. In many cases a small parameter appears in a natural way and, thence, the whole flow field can be split into partially overlapping regions where much simpler solutions become valid.

The structure of the inner core, far from the free surface of the floating zone, is well known in many cases. The known mathematical solution to this problem is matched, to a given order in the small parameter, with the solution, valid up to this order, in a region close to the free surface. To do so the technique of

†See Carruthers and Grasso (1972), and Martínez Herranz (1976, 1977). 
matched asymptotic expansionst can be used. Even in the cases in which the solution on a given zone cannot be expressed in closed form, the matching conditions give some insight into the main characteristics of the flow field.

The problem of spin-up from the rest of an initially cylindrical zone is considered here with some detail. The undisturbed zone is held between two parallel coaxial discs having equal diameters. The contact angle between liquid, disc material and surrounding atmosphere is assumed to be smaller than $90^{\circ}$, so that, given the disc diameter and the distance between the discs, there is a fixed liquid volume which keeps cylindrical the undisturbed floating zone. Suddenly one of the discs is set impulsively into rotating motion and a shear layer near the disc starts to thicken by viscous diffusion.

As it has been indicated above, we will look for a solution of the fluid motion that is close to the exact configuration at rest, thence this solution will hold only for small values of the time elapsed since the starting of the process. The mathematical technique used is fairly general, and some other configurations where a small parameter appears could be dealt with on similar grounds.

Spin-up from the rest has been considered in the past for both laterally unbound liquids (Nigam, 1951; Benton, 1966), and for liquids within a cylindrical container, $\ddagger$ but no similar analysis for the case of containment by surface tension seems to exist.

This work is part of a more general endeavor for predicting the behavior of a floating zone subjected to mechanical disturbances in a reduced gravity environment (ESA experiment E-67, 1st Spacelab Mission). The aim of any pre-flight work of this kind would be to pave the way to a sound evaluation of the "unexpected" results which could arise from the space experiment.

Once the problem discussed here has been considered with some detail, one can question whether this particular configuration could be experimentally simulated on the ground laboratory or not. Although the answer is affirmative in this particular case, as we will show later, other elusive problems, such as those concerning the structure of the shear layer between unequally rotating discs, whose experimental simulation requires large floating zones, cannot be simulated so easily.

\section{Rotating liquids contained by surface tension}

\section{Basic equations}

The diagram in Fig. 1 shows the geometry, coordinate system and fluid velocity components for a spinning floating zone. The radius of the column is $R$, whereas the zone length is let unspecified since it does not appear in the problem which concerns the flow field near one of the discs.

The nondimensional differential equations for the axisymmetric rotating flow in cylindrical coordinates are (Greenspan, 1968):

$$
\frac{\partial u}{\partial t}+u \frac{\partial u}{\partial r}-\frac{v^{2}}{r}+w \frac{\partial u}{\partial z}=-\frac{\partial p}{\partial r}+E\left(\frac{\partial^{2} u}{\partial r^{2}}+\frac{1}{r} \frac{\partial u}{\partial r}+\frac{\partial^{2} u}{\partial z^{2}}-\frac{u}{r^{2}}\right),
$$

tSee Cole (1968), Eckhaus (1973), Nayfeh (1973) and Van Dyke (1975).

$\ddagger$ See Wedemeyer (1964), Watkins and Hussey (1973) and Weidman (1976). 


$$
\begin{gathered}
\frac{\partial v}{\partial t}+u \frac{\partial v}{\partial r}+\frac{u v}{r}+w \frac{\partial v}{\partial z}=E\left(\frac{\partial^{2} v}{\partial r^{2}}+\frac{1}{r} \frac{\partial v}{\partial r}+\frac{\partial^{2} v}{\partial z^{2}}-\frac{v}{r^{2}}\right) \\
\frac{\partial w}{\partial t}+u \frac{\partial w}{\partial r}+w \frac{\partial w}{\partial z}=-\frac{\partial p}{\partial z}+E\left(\frac{\partial^{2} w}{\partial r^{2}}+\frac{1}{r} \frac{\partial w}{\partial r}+\frac{\partial^{2} w}{\partial z^{2}}\right) \\
\frac{1}{r} \frac{\partial}{\partial r}(r u)+\frac{\partial w}{\partial z}=0
\end{gathered}
$$

To write down these equations any length has been made dimensionless with $R$, any velocity with $\Omega R$, where $\Omega$ is the maximum angular velocity prevailing in the flow field (either $\Omega_{w}$ or $\Omega_{f}$ ). Time has been made dimensionless with $\Omega^{-1}$, and guage pressure with $\rho \Omega^{2} R^{2}$. Finally, $E$ is the Ekman number

$$
E=\frac{\nu}{\Omega R^{2}}
$$

The boundary conditions at the disc are:

$$
v=r \frac{\Omega_{w}(t)}{\Omega}, \quad u=w=0 \quad \text { on } z=0 .
$$

Far from the disc,

$$
\lim _{z \rightarrow \infty}\left(v-r \frac{\Omega_{f}(t)}{\Omega}\right)=0, \quad \lim _{z \rightarrow \infty} u=0 .
$$

The vertical velocity component cannot be prescribed at infinity because the viscous boundary layer at the plate induces an axial flux which persists throughout the flow field.

\section{Boundary conditions at the free surface}

The diagram in Fig. 2 sketches the free surface, which bounds laterally the floating zone. The equation of this surface, in dimensionless form, is:

$$
r=1+l(z, t) .
$$

The first boundary condition will express the balance of normal forces at the interface. These forces are (Landau and Lifshitz, 1959), the capillary pressure (as given by Laplace's formula), the excess of the local pressure over the ambient pressure, and the normal viscous stresses. In dimensionless form:

$$
p-\frac{2 E}{1+\left(\frac{\partial l}{\partial z}\right)^{2}}\left[\frac{\partial u}{\partial r}+\left(\frac{\partial l}{\partial z}\right)^{2} \frac{\partial w}{\partial z}-\frac{\partial l}{\partial z}\left(\frac{\partial w}{\partial r}+\frac{\partial u}{\partial z}\right)\right]=\frac{1}{C\left[1+\left(\frac{\partial l}{\partial z}\right)^{2}\right]^{3 z}}\left[\frac{1+\left(\frac{\partial l}{\partial} \frac{z}{z}\right)^{2}}{1+l}-\frac{\partial^{2} l}{\partial z^{2}}\right]
$$




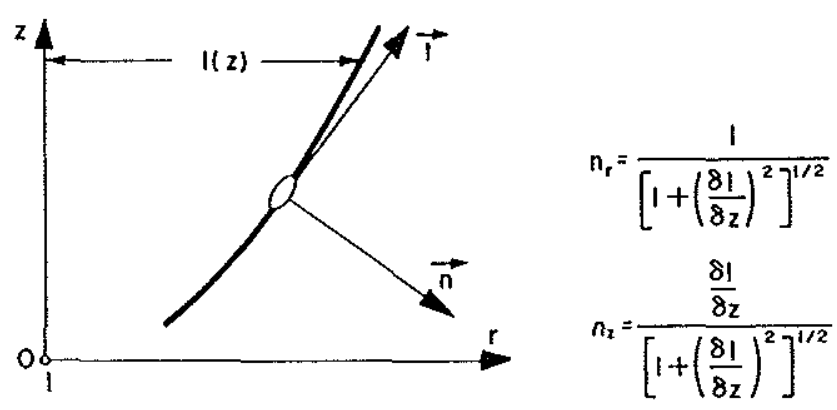

Fig. 2. Sketch of a free surface element in the $\theta$ plane.

where $C$ is the dimensionless rotation parameter (Bond number in terms of the centrifugal acceleration $R \Omega^{2}$ ),

$$
C=\frac{\rho R^{3} \Omega^{2}}{\sigma}
$$

Two more boundary conditions are deduced by expressing that the tangential viscous stresses vanish at the interface. That is to say, that: $T \cdot n-$ $(n \cdot T \cdot n) \cdot n=0, T$ being the viscous stress tensor and $n$ the unit normal to the perturbed surface. In dimensionless form these conditions become:

1. For the axial component,

$$
2 \frac{\partial l}{\partial z}\left[\frac{\partial u}{\partial r}-\frac{\partial w}{\partial z}\right]+\left[1-\left(\frac{\partial l}{\partial z}\right)^{2}\right]\left[\frac{\partial w}{\partial r}+\frac{\partial u}{\partial z}\right]=0
$$

2. For the azimuthal component,

$$
\frac{\partial v}{\partial r}-\frac{v}{r}-\frac{\partial l}{\partial z} \frac{\partial v}{\partial z}=0
$$

On the other hand, the condition that the interface is a fluid surface, since the mass flow rate through it is zero, yields:

$$
\frac{\partial l}{\partial t}-u+w \frac{\partial l}{\partial z}=0
$$

Furthermore, it is assumed that the free surface remains anchored to the disc edge, thus $l(0, t)=0$. This assumption seems to be substantiated by the experimental evidence, at least for moderate spinning rates. The spreading of the liquid column along the end discs poses interesting problems which have been the subject of some attention (Hocking, 1976 and 1977).

\section{Spin-up of a floating zone from rest}

The direct analytical approach to time dependent problems of rotating liquids contained by surface tension is difficult because of the non-linearity of the 
governing differential eqns (1) and boundary conditions (2-4). Thence, we will resort to linearization methods by slightly disturbing some exact solution and exploring the consequences of the disturbance. In the present case the "exact solution" is that corresponding to a cylindrical floating zone in equilibrium at rest. The perturbation consists of suddently starting to spin one of the end plates about the axis of rotation with constant angular velocity. Formal series expansion for velocity and pressure in a small parameter $\epsilon$, which measures the time from starting, will be introduced into the full Navier-Stokes equations and boundary conditions. Since the expansion must hold for arbitrary small values of $\epsilon$, terms of like orders in $\epsilon$ must separately satisfy each equality, thus a relatively simple set of linear ordinary differential equations with variable coefficients and non-linear forcing functions results. These equations are uncoupled and they can be solved exactly in closed form. In practice, however, two difficulties prevent the calculation of higher order terms, namely:

1. The complexity of the forcing function increases very rapidly with order in the set, and

2. The perturbation scheme breaks-down in the proximity of the interface. Before pursuing further we are forced to examine the details of the flow in the corner region where several fluid variables experiene sharp changes.

Both solutions, that in the central core and that in the corner region, complement each other, one being valid in the region where the othe $r$ fails, and both must be matched in an intermediate region of common validity.

In the present case only first order terms have been obtained, and even in a not completely satisfactory way. This is a fairly modest achievement if one realizes that, judging by the case of the laterally unbound fluid real $\mathrm{m}$, the first order solution correspond to no more than the first-half radian of the disc motion, whereas the steady state is approached after about 2 radians of motion. It is, nevertheless, our feeling that this solution represents a start in the problem and an indication of the way to obtain more complete solutions.

\section{The central core}

It is only logical to assume that there is a central core which, at least to first order, does not feel the effects of the free boundary. The solution for this central core will be that corresponding to the motion of a viscous fluid, confined to the upper half plane $(z \geq 0)$, when at some initial instant the disc, of infihite radius, starts spinning with constant angular velocity.

The viscous shear layer produced near the disc is, at the very beginning, very thin. Thence, aiming at the use of coordinates of order unity, we introduce a magnified time, $\tau$, and a magnified distance to the disc, $\eta$, whereas $r$ is left unaltered.

$$
\tau=\frac{t}{\epsilon}, \quad \eta=\frac{z}{2 \sqrt{ }(a E \epsilon \tau)}
$$

The asymptotic solution to this problem, as the parameter $\epsilon$ becames small, can be written down as follows: 


$$
\begin{aligned}
u(r, z, t) & =\epsilon \tau r\left[f_{1}(\eta)+(\epsilon \tau)^{2} f_{2}(\eta)+(\epsilon \tau)^{4} f_{3}(\eta)+\ldots\right] \\
v(r, z, t) & =r\left[g_{1}(\eta)+(\epsilon \tau)^{2} g_{2}(\eta)+(\epsilon \tau)^{4} g_{3}(\eta)+(\epsilon \tau)^{6} g_{4}(\eta)+\ldots\right], \\
w(z, t) & =-4 \epsilon \tau \sqrt{ }(E \epsilon \tau)\left[h_{1}(\eta)+(\epsilon \tau)^{2} h_{2}(\eta)+(\epsilon \tau)^{4} h_{3}(\eta)+\ldots\right], \\
p(z, t)-\frac{1}{C} & =2 E \epsilon \tau\left[p_{1}(\eta)+(\epsilon \tau)^{2} p_{2}(\eta)+(\epsilon \tau)^{4} p_{3}(\eta)+\ldots\right] .
\end{aligned}
$$

This expansion scheme is due to Benton (1966). The functions $f_{1}, g_{1}$ and $h_{1}$ were found in closed form by Thiriot (1940) (quoted by Benton). $p_{1}$ was also given in closed form by Nigam (1951). Benton found $g_{2}$ in closed form and $f_{2}, f_{3}$, $g_{3}, g_{4}, h_{2}$ and $h_{3}$ by numerical integration. All these functions are tabulated in Benton's paper. Some of them have been plotted in Fig. 3.

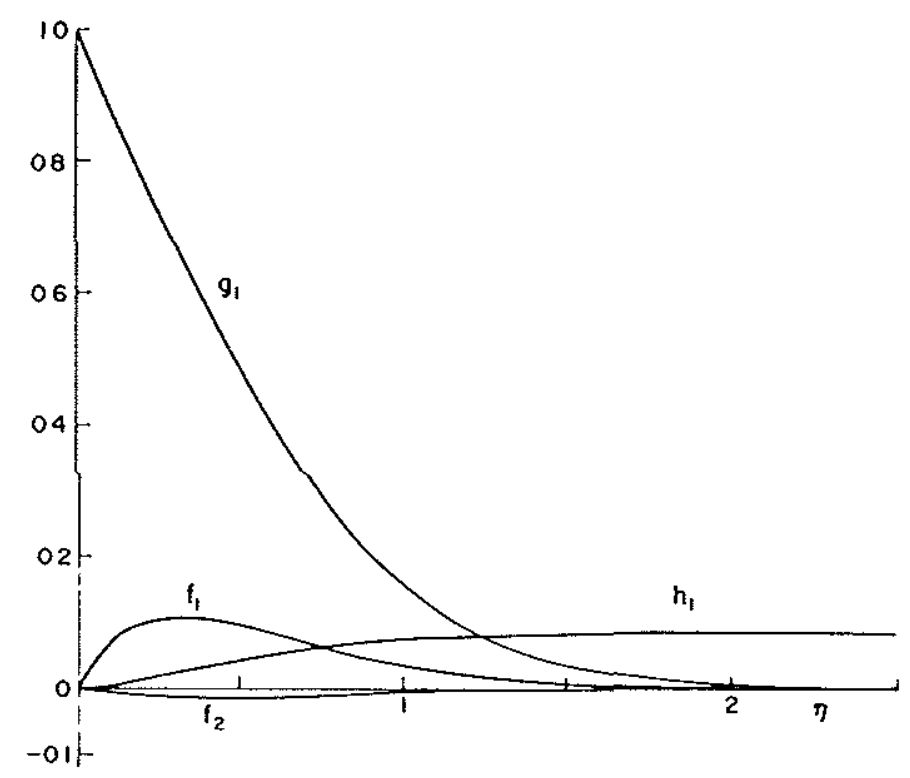

Fig. 3. The functions $f_{1}, f_{2}, g_{1}$ and $h_{1}$ as given by Benton (1966).

The corner region

We suspect that the central core solution is not valid near the free surface, where very complicated boundary conditions must be fulfilled. It is hoped, however, that the region where the core solution is non-uniformly valid is very thin, and this suggests using the following "corner region variables" (Fig. 4).

$$
\tau=\frac{t}{\epsilon} ; \quad \eta=\frac{z}{\sqrt{ }(E \epsilon \tau)} ; \quad \xi=\frac{r-1}{2 \sqrt{ }(E \epsilon \tau)}
$$

The asymptotic expansions for the relevant variables in this zone are:

$$
\begin{gathered}
u(r, z, t)=\epsilon \tau\left[\underline{r f_{1}(\eta)}+r^{-1} U_{1}(\xi, \eta)+2 \sqrt{ }(E \epsilon \tau) U_{2}(\xi, \eta)+\ldots\right], \\
v(r, z, t)=r g_{1}(\eta)+\ldots,
\end{gathered}
$$




$$
\begin{gathered}
w(r, z, t)=\epsilon \tau\left[r^{-1} W_{1}(\xi, \eta)+2 \sqrt{ }(E \epsilon \tau) W_{2}(\xi, \eta)-\underline{4 V(E \epsilon \tau) h_{1}(\eta)}+\ldots\right], \\
p(r, z, t)-\frac{1}{C}=\sqrt{ }(E \epsilon \tau)\left[\frac{1}{2} P_{1}(\xi, \eta)+\sqrt{ }(E \epsilon \tau) P_{2}(\xi, \eta)+\underline{2 \sqrt{ }(E \epsilon \tau) p_{1}(\eta)}+\ldots\right], \\
l(z, t)=(\epsilon \tau)^{2}\left[L_{1}(\eta)+2 \sqrt{ }(E \epsilon \tau) L_{2}(\eta)+\ldots\right]
\end{gathered}
$$

Underlined terms are those entering into the central core solution. $r$ has been used as far as possible to simplify writing. It is to be noted that these expansions proceed in powers of $(\epsilon \tau)^{1 / 2}$ and, thence, several terms must be calculated for each term of the central core expansion.

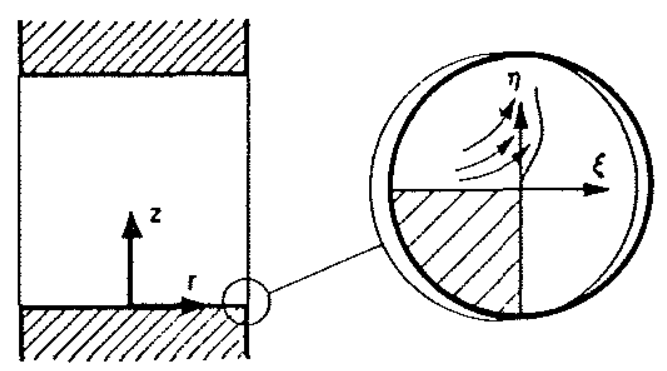

Fig. 4. Corner region variables.

Substituting expansions (6) into eqns (1) and boundary conditions (2-4) and realizing that the problem for the azimuthal velocity, $v$, remains uncoupled, one gets the basic equations, defining the first and second order "corner region problems", given in Table 1. Both problems are two dimensional and linear. Unfortunately they are boundary value elliptic problems and attempts made to found analytical simple solutions meet, up to this moment, with partial success only.

It should be noticed that since the radial velocity can be calculated to first and second order without resorting to pressure, the shape of the free surface is independent of the Bond number, $C$, provided that it is (in addition to constant) of order unity or larger. Values of $C^{-1}$ for several liquids in a typical case are given in Table 2.

\section{Several results}

The first order problem has been solved close to the corner (small values of $\rho$ ) and far from it (large "magnified" values of $\rho$ ).

The stream function for small $\rho$ has been found to be,

$$
\psi(r, z, t)=\epsilon \tau\left[\frac{\rho^{4}}{4 !} H_{4}(\theta)+\frac{\rho^{5}}{5 !} H_{5}(\theta)+\frac{\rho^{6}}{6 !} H_{6}(\theta)+\ldots\right],
$$

where $\rho^{2}=\xi^{2}+\eta^{2}, \theta=\tan ^{-1} \xi / \eta$, and

$$
H_{4}(\theta)=3 \frac{\mathrm{d}^{3} f_{1}}{\mathrm{~d} \eta_{\eta=0}^{3}} \cos ^{2} \theta\left(1-\frac{5}{6} \cos ^{2} \theta\right)
$$




$$
\begin{aligned}
& H_{5}(\theta)=\frac{10}{3} \frac{\mathrm{d}^{4} f_{1}}{\mathrm{~d} \eta_{\eta=0}^{4}} \cos ^{3} \theta\left(1-\frac{9}{10} \cos ^{2} \theta\right), \\
& H_{6}(\theta)=\frac{15}{2} \frac{\mathrm{d}^{5} f_{1}}{\mathrm{~d} \eta_{\eta=0}^{5}} \cos ^{2} \theta \sin ^{2} \theta \cos 2 \theta .
\end{aligned}
$$

This stream function depicts the fluid motion devoided of the azimuthal velocity component.

Several streamlines have been plotted in Fig. 5. These streamlines change with time. The fact that they impinge normally to the undisturbed free surface indicates that the fluid set into radial motion by centrifugal forces feeds the fluid domain which grows because of the free surface displacement.

It is also interesting to point out that the curvature of the streamlines is moderate in any case and seems to reach its largest value in the proximity of the corner where the velocity is small, thence the pressure gradients developed in the proximity of the free surface are not large.

Table 1. First and second order problems for the corner region

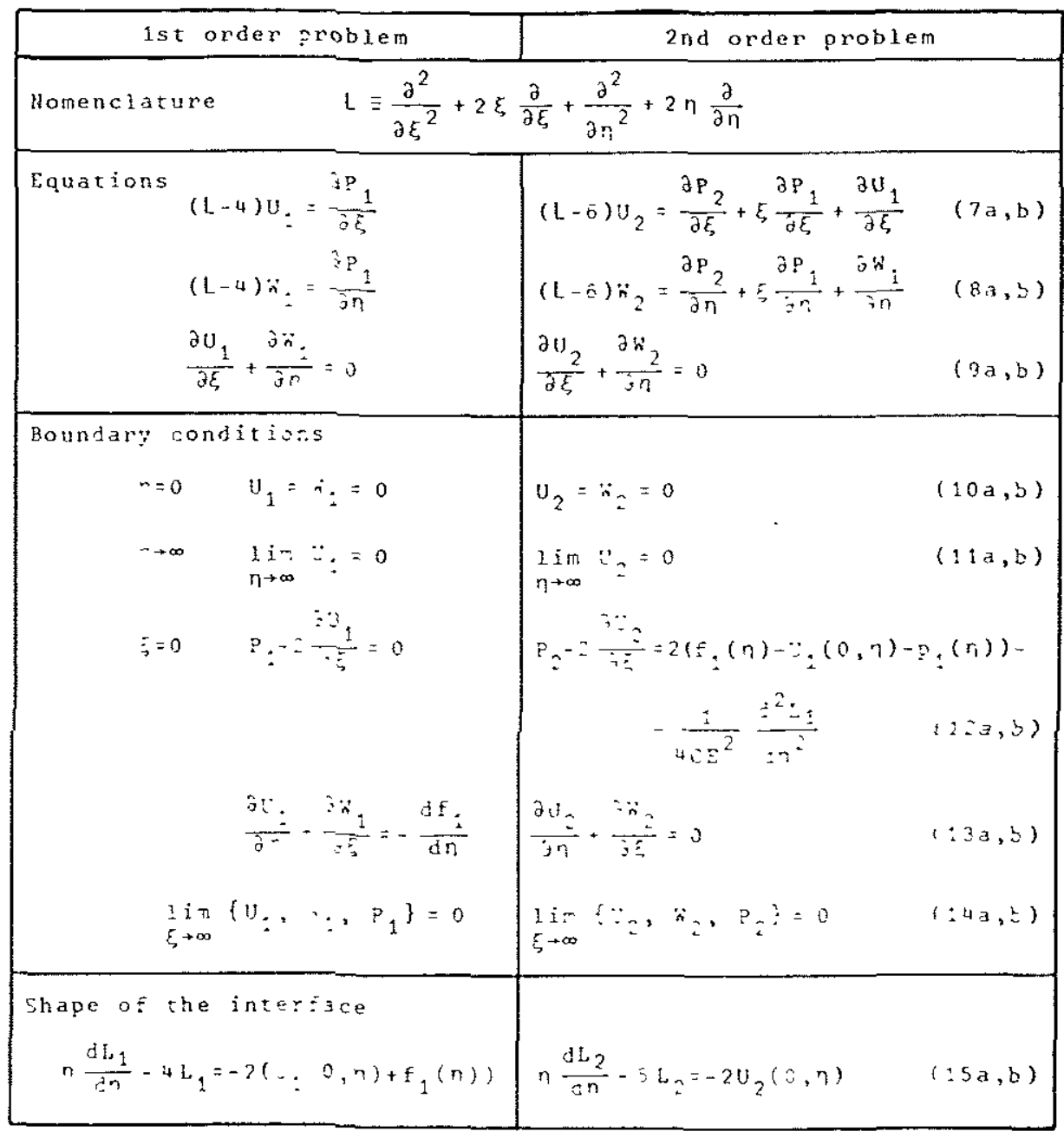


Table 2. Values of the reciprocal of the bond number for several liquids

\begin{tabular}{|c|c|c|c|c|}
\hline Liquid & {$\left[\begin{array}{c}\rho \times 10^{-3} \\
{\left[\mathrm{~kg} \cdot \mathrm{m}^{-3}\right]}\end{array}\right.$} & {$\left[\begin{array}{c}\sigma \times 10^{3} \\
{\left[N \cdot m^{-1}\right]}\end{array}\right.$} & $\begin{array}{l}\text { References } \\
\text { and Comments }\end{array}$ & $c^{-1}$ \\
\hline Water & .9982 & 72.583 & \multirow{4}{*}{$\begin{array}{l}\text { Riddick } E \\
\text { Bunger }(1970)\end{array}$} & .582 \\
\hline Propylene Glycol & 1.0328 & 72 & & .558 \\
\hline Gl;cerol & 1.2613 & 63.3 & & .401 \\
\hline Diethylene Glyc. 1 & 1.1164 & 45.21 & & .324 \\
\hline $\begin{aligned} \text { Dite ethyl Silicone } \\
1000 \mathrm{CS}\end{aligned}$ & .973 & 21.2 & \multirow{2}{*}{$\begin{array}{l}\text { DOW CORNIHG } \\
(19633)\end{array}$} & .174 \\
\hline $\begin{array}{c}\text { Dimethyl silicone } \\
5 \mathrm{cs}\end{array}$ & .918 & 19.7 & & .172 \\
\hline $\begin{array}{l}\text { Water contaminated with } \\
\text { n-tropanol }\end{array}$ & .9982 & 70.40 & \multirow{3}{*}{$\begin{array}{l}250 \mathrm{ml} \text { of water } \\
\text { with } 1 \text { drop of } \\
\text { contaminant. } \\
\text { Padday (1977). }\end{array}$} & .564 \\
\hline $\begin{array}{l}\text { Wacer contaminated with } \\
\text { Tricresyl Phosphate }\end{array}$ & .95 & 63.30 & & .507 \\
\hline $\begin{array}{l}\text { "Uater contaminated with } \\
\text { Eletiyl Phthalate }\end{array}$ & .9982 & 49.80 & & .399 \\
\hline
\end{tabular}

The distribution of radial velocity, $U_{1}(\xi, \eta)+f_{1}(\eta)$, near the disc is shown in Fig. 6. This distribution, which will present an inflection point outside the frame of the figure, reminds that existing near the separation point of a boundary layer. This resemblance is not complete, since the non-linear momentum terms, which play an important role in boundary layer separation, are negligible here. One must say that the existence of the mentioned inflection points strongly indicates the possibility of instabilities originating near the disc.

Figure 7 shows the radial velocity, $U_{1}(0, \eta)+f_{1}(\eta)$, across the boundary $\xi=0$, as well as the shape, $L_{1}(\eta)$, of the free surface.

Since we were unable to calculate the velocity field except for extreme values

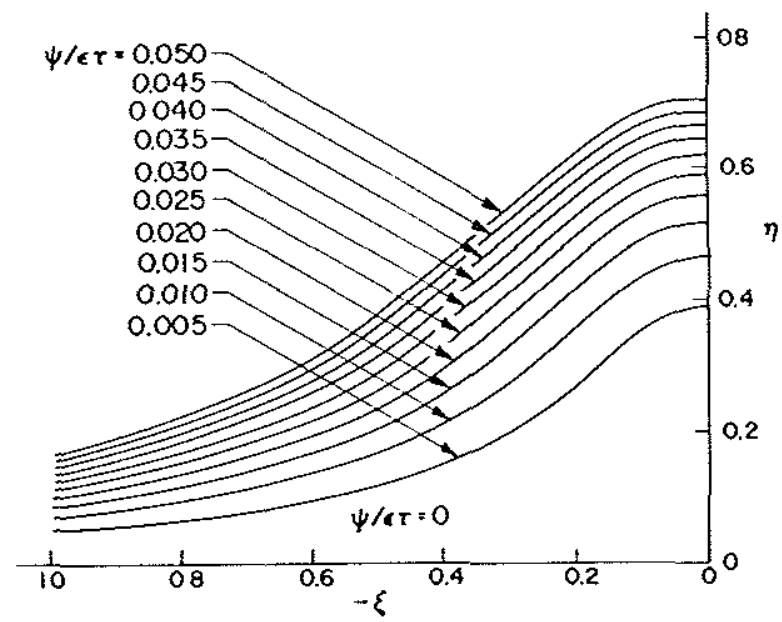

Fig. 5. The streamlines near the corner of the floating zone as calculated by means of eqns (16) and (17). 


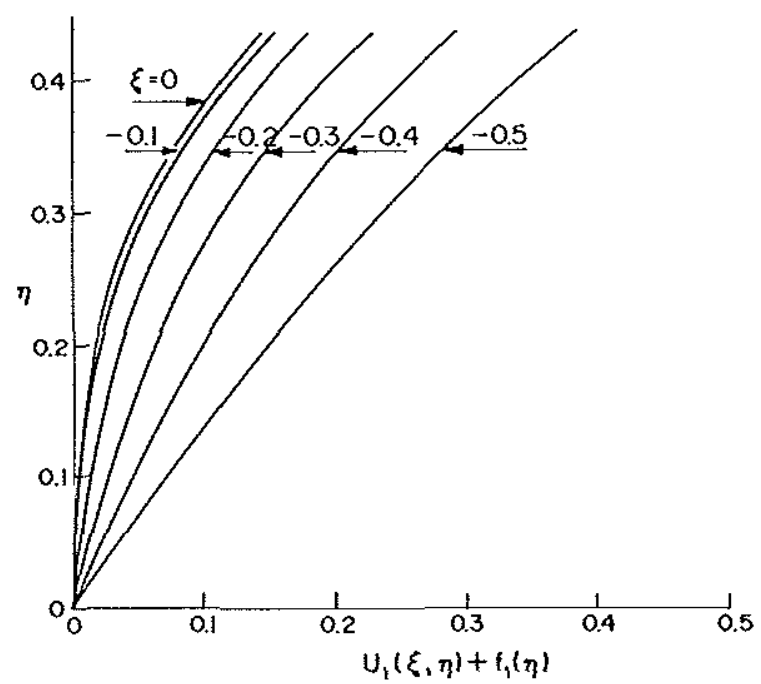

Fig. 6. Radial velocity distribution near the disc.

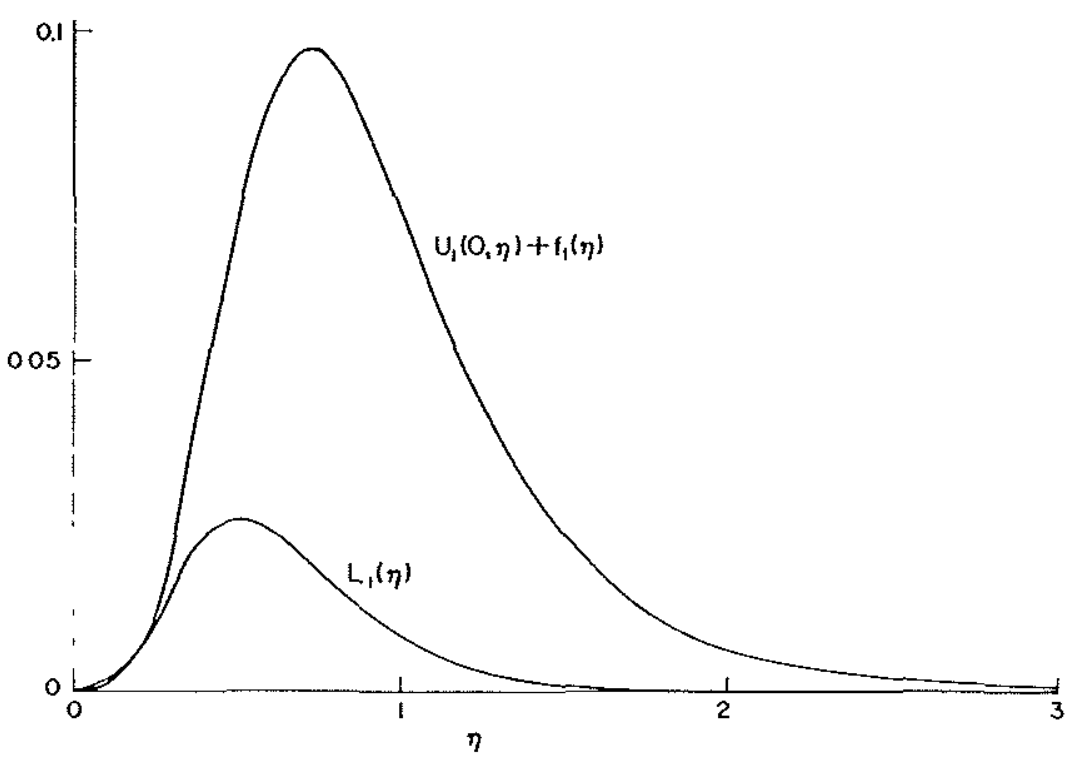

Fig. 7. Radial velocity across the boundary $\xi=0$ ard shape of the free surface.

of $\eta$, we resorted to a patching between the function giving $U_{1}(0, \eta)$ for small and for large values of $\eta$. This was done as follows:

First a solution of the problem valid near $\xi=0$ was sought in the form:

$$
\begin{aligned}
& U_{1}(\xi, \eta)=\sum_{0}^{\infty} \frac{\xi^{2 m}}{2 m !} U_{2 m}(\eta), \\
& \left.\begin{array}{l}
W_{1}(\xi, \eta) \\
P_{1}(\xi, \eta)
\end{array}\right\}=\sum_{0}^{\infty} \frac{\xi^{2 m+1}}{(2 m+1) !}\left\{\begin{array}{l}
W_{2 m+1}(\eta) \\
P_{2 m+1}(\eta)
\end{array}\right.
\end{aligned}
$$


Substitution of these expansions into the equations of motion (7a)-(9a), with boundary conditions (12a) and (13a), gives a set of ordinary differential equations in $\eta$. The successive stages are not uncoupled however, rather there is at each stage more unknowns than equations. This is so because the problem is elliptic and, thence, the flow near the free surface is affected by that farther from the surface. Nevertheless, it is possible (van Dyke, 1966) to break the chain at some stage, leading to a determinate set of equations. The simplest approach, in our case, consists in assuming that pressure gradients can be neglected throughout so that the following expression results for the radial velocity:

$$
\begin{array}{r}
U_{1}(0, \eta)+f_{1}(\eta)=\frac{\pi}{2}\left\{F_{1}(\eta) \int_{\eta}^{\infty} \mathrm{e}^{x^{2} / 2} \operatorname{erfc}^{2} x F_{2}(x) \mathrm{d} x-F_{2}(\eta) \int_{\eta}^{\infty} \mathrm{e}^{x^{2} / 2} \operatorname{erfc}^{2} x F_{1}(x) \mathrm{d} x\right\} \\
+A F_{2}(\eta),
\end{array}
$$

with

$$
\begin{aligned}
& F_{1}(\eta)=1+\eta^{2} \\
& F_{2}(\eta)=\left(1+\eta^{2}\right) \operatorname{erfc} \frac{\eta}{\sqrt{ } 2}-\sqrt{\left(\frac{2}{\pi}\right) \eta \mathrm{e}^{\left(-\eta^{2} / 2\right)}}
\end{aligned}
$$

Although one is tempted to calculate $A$ by expressing $U_{1}(0,0)=0$, this is not advisable, because the uniform pressure assumption is only valid far from the plate. On the contrary, near the plate the following expression holds:

$$
U_{1}(0, \eta)+f_{1}(\eta)=\frac{1}{12} \frac{\mathrm{d}^{3} f_{1}}{\mathrm{~d} \eta_{\eta=0}^{3}} \eta^{3}+\ldots
$$

Then a composite function was written down, and $A$ was calculated through the requirement of overall mass preservation as follows:

$$
\int_{0}^{\infty}\left\{U_{1}(0, \eta)+f_{1}(\eta)\right\} \mathrm{d} \eta=\lim _{\eta \rightarrow \infty} h_{1}(\eta)
$$

Recall that $4 \epsilon \tau \sqrt{ }(E \epsilon \tau) \pi\left\{\lim _{\eta \rightarrow \infty} h_{1}(\eta)\right\}$, eqn (5c), gives, as a first approximation, the mass flow rate towards the plate which replaces the liquid propelled outwards in the boundary layer.

Once an approximation for $U_{1}(0, \eta)$ has been obtained, $L_{1}(\eta)$ is deduced from eqn (15a). The resulting curve is also given in Fig. 7.

It can be seen in Fig. 7 that the floating zone remains cylindrical in the close proximity of the plate. This contrasts with most available information on the shape of rotating floating zones, which seems to indicate that some conicalness exists near the plates. This conicalness may be due either to gravitational effects or to later developments in the rotating motion. In fact the boundary condition (3b) shows that provided that $\partial l / \partial z$ is small enough, as happens to be the case in the first stages of the rotating motion development, $\partial w / \partial r=-\partial u / \partial z$. Since near 
the plate $\partial w / \partial r=0, u(0, \eta)$ has a zero at least of order $\eta^{2}$ when $\eta=0$. Thence the free surface must remain cylindrical near the end disc, (see eqns $15 a, b$ ).

\section{Other central core solutions}

The approach pursued here is based on the existence of an exact solution which is slightly disturbed. The same approach can be surely used in other cases of interest.

A list of solutions, already available, which could be relevant to problems connected with the structure of rotating floating zones is given in Table 3 . In most cases solutions for not too small values of the controlling parameter exist, but they are of little use here since the boundary conditions at the free surface are only simple when that surface departs slightly from a circular cylinder.

The free shear layer under equal counter-rotation (case 6 in Table 3), has been included because its study requires only small amendments to the near-thedisc solution. A more difficult problem concerns the location of that shear layer. In the case of exactly equal counter-rotation of the discs the shear layer is

Table 3. Relevant candidate central core solutions

\begin{tabular}{|c|c|c|c|c|}
\hline \multicolumn{3}{|c|}{ Boundary Conditions } & \multirow{2}{*}{$\begin{array}{c}\text { Small } \\
\text { Parameter }\end{array}$} & \multirow{2}{*}{ References } \\
\hline & at $t<0$ & at $t \geq 0$ & & \\
\hline 1 & $\frac{i j \Omega_{\mathrm{f}}}{\frac{i}{\text { (i) } 3 \Omega_{\mathrm{f}}}}$ & $\dot{\psi}^{p \Omega_{\mathrm{f}}}$ & $\varepsilon=\frac{\Omega_{H}}{\Omega_{f}}-1$ & $\begin{array}{l}\text { Greenspan, } \\
1968, \text { pp. 28-34 }\end{array}$ \\
\hline & 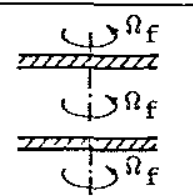 & 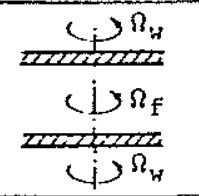 & $\varepsilon=\frac{\Omega_{i-j}}{\Omega_{f}}-1$ & $\begin{array}{c}\text { Greenspan and Howard, } \\
1963\end{array}$ \\
\hline 3 & 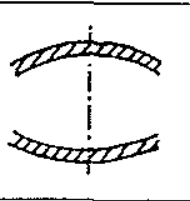 & 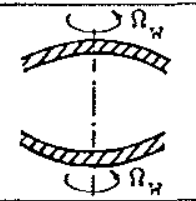 & $\varepsilon=\sqrt{E}$ & $\begin{array}{l}\text { Greenspan, } \\
1968, p p \cdot 38-63\end{array}$ \\
\hline 4 & $\frac{1}{i}^{\Omega_{f}}$ & $\frac{\underbrace{\Omega_{f}}}{v_{i}+\Omega_{w}}$ & $\varepsilon=\frac{t}{\tau}$ & $\begin{array}{c}\text { Davis and L,udford, } \\
1974-a .\end{array}$ \\
\hline 5 & 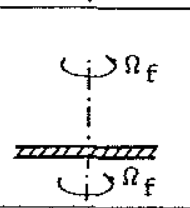 & $\begin{array}{c}\dot{j} \Omega_{\mathrm{f}} \\
\vdots \\
\frac{1}{1}\end{array}$ & $\varepsilon=\frac{t}{T}$ & $\begin{array}{c}\text { Davis and Ludford, } \\
1974-b .\end{array}$ \\
\hline 6 & $-\frac{i}{i}+-\cdot--$ & 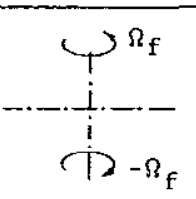 & $\varepsilon=\frac{t}{T}$ & \\
\hline
\end{tabular}


obviously placed midway between both discs. Other cases are difficult to elucidate. Further, Mellor et al. (1968), showed that several cells in the meridian plane could appear. It should be said, in addition, that to correctly locate these layers requires the knowledge of higher order approximations which are, at present, far from available.

\section{The feasibility of ground-based experiments}

Floating liquid zones can be simulated on the surface of the earth by use of the "neutral buoyancy" (Plateau), technique. This technique involves the suspension of one liquid inside another with which it is immiscible but precisely equal in density. The neutral buoyancy technique has been used in floating zones by several experimenters. $t$ Their results are relevant to the stability of floating zones under disturbances akin to those which would appear in the space experiment. The main objection to this technique lies in the presence of both pressure and viscous forces, induced by the movement of the outer liquid, which hide some dynamical effects. In our particular case, viscous stresses acting at the interface between both liquids would invalidate the boundary conditions ( $3 \mathrm{~b}$ ) and (3c).

Fortunately, most of the effects associated to the spin-up arise near the plates and, thence, their simulation requires zones of short length.

Figure 8 shows the maximum stable length of a floating zone in a graviational field, $2 h$, vs the radius, $R$, of the zone. Notice that for large values of $R$ (in the case of water for $R>6 \mathrm{~mm})$ an asymptotic value, $2 h=2.84 \mathrm{~V}(\sigma / g \rho)$, is reached. Values of $2 h$ for several liquids, and large values of $R$, are given in Table 4 .

Let us compare these lengths with the thicknesses of the viscous boundary layers, close to the discs, which were previously analyzed.

As can be seen in Fig. 3, the fluid dynamic phenomena of interest turn up

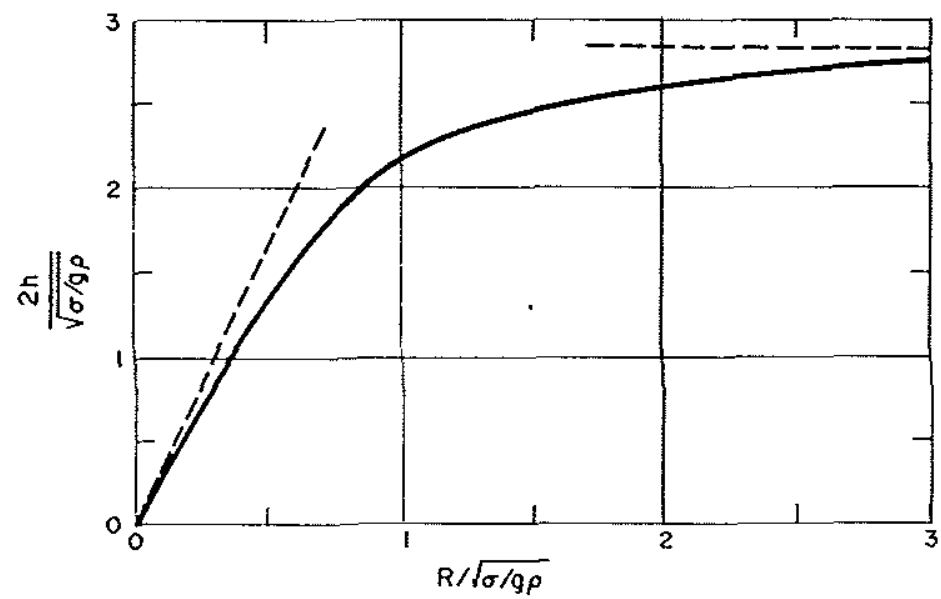

Fig. 8. Maximum stable length of a floating zone in a gravitational field, according to Heywang (From Wuest, 1976).

†See Plateau (1859), Mason (1970) and Carruthers and Grasso (1972) among others. 
Table 4. Maximum stable length, $2 h$, of a floating zone, according to Heywang asymptotic formula, compared to twice the thickness, $2 z$, of the viscous layer near the end disc. $\Omega=1 \mathrm{rad} . \mathrm{s}^{-1}$. Liquids at temperature close to $300 \mathrm{~K}$

\begin{tabular}{|c|c|c|c|c|}
\hline Liquid & $\begin{array}{l}\sigma / g \rho \times 10^{6} \\
g=9.81 \mathrm{~m} . \mathrm{m}^{2}{ }^{-2}\end{array}$ & $\begin{array}{c}\nu \times 10^{6} \\
{\left[\mathrm{~m}^{2} \cdot \mathrm{s}^{-1}\right]}\end{array}$ & $\begin{array}{c}2 \mathrm{~h} \times 10^{3} \\
{[\mathrm{~m}]}\end{array}$ & $\begin{array}{c}2 \mathrm{z} \times 10^{3} \\
{[\mathrm{~m}]}\end{array}$ \\
\hline Water & 7.41 & 1.0037 & 7.73 & 8.01 \\
\hline Propylene Glycol & 7.11 & 54.23 & 7.57 & 58.9 \\
\hline Glycarol & 5.12 & 487.6 & 6.42 & 176. \\
\hline Diethylene Glycol & 4.12 & 31.98 & 5.77 & 45.2 \\
\hline $\begin{array}{r}\text { Dimethy } \mathrm{Silicone} \\
1000 \mathrm{Cs}\end{array}$ & 2.22 & 1000 & 4.23 & 253. \\
\hline $\begin{array}{r}\text { Dimethyl Silicone } \\
5 \mathrm{cs}\end{array}$ & 2.19 & 5. & 4.20 & 17.9 \\
\hline
\end{tabular}

within dimensionless distances to the end discs which are at most $\eta=2$. This means in the physical variables $z \leq 4 R V(E \epsilon \tau)$. Furthermore, our approach is based on the hypothesis that $\epsilon \tau$ is small $(\epsilon \tau \leq 1)$. Values of $2 z=8 \mathrm{~V}(v / \Omega)$, are also given in Table 4 for the same liquids and conditions as stated in Table 2 above.

One can realize from Table 4 that both lengths, $2 h$ and $2 z$, are very similar in the case of water. For more viscous liquids, the viscous boundary layer thickens very rapidly. To avoid this fast thickening, the spinning velocity $\Omega$ could be increased, with the subsequent decrease in the experimental time.

An apparatus to simulate the spin-up from the rest of a floating zone, which is based on the above ideas, has been constructed in our laboratory. The results which have been obtained up to this moment are not conclusive enough to be presented here.

\section{Conclusion}

The work presented above is incomplete and a more detailed study should be made. Nevertheless it is felt that this work could introduce the analysis of several phenomena which appear in a spinning floating zone near the discs.

Although the fluid domain which has been considered up to now is fairly reduced on a volumetric basis, it is the more interesting from both the fundamental point of view and from the applications.

From the fluid dynamic point of view the corner region is the source of instabilities which should be considered with detail in the future. Furthermore, in the crystal growth technique special care must be paid to the growth front (that is to say, the zone near the end disc), when aiming at the obtention of homogeneous single crystals.

A more complete study of the boundary layer near the disc would include: non-uniform temperature fields, highly temperature dependent material properties (particularly viscosity), and phase changes. It is our impression that all these effects would be considered at not excessive extra work, once the incompressible problem has been correctly solved. 


\section{References}

Benton, E. R. (1966) On the flow due to a rotating disk. J. Fluid Mech. 24, 781-800.

Carruthers, J. R. and Grasso, M. (1972) Studies of floating liquid zones in simulated zero gravity. $J$. Appl. Phys. 43, 436-445.

Carruthers, J. R., Gibson, E. G., Klett, M. G. and Facemire, B. R. (1975) Studies of rotating liquid floating zones on skylab IV, AIAA Paper 75-692.

Cole, J. D. (1968) Perturbation Methods in Applied Mathematics. Blaisdell, Waltham, Mass., 120-221.

Chang, C. E. and Wilcox, W. R. (1976) Analysis of surface tension driven flow in floating zone melting. Int. J. Heat Mass Transfer 19, 355-366.

Chun, C. H. and Wuest, W. (1977) Flow phenomena in gravitationless melting zones in the presence of electromagnetic fields, Presented at 20th COSPAR Meeting, Tel-Aviv, Israel, 7-18 June, 1977.

Davis, R. W. and Ludford, G. S. S. (1974a) Stagnation-point flow in a rotating fluid. Phys. Fluids 17, $275-279$.

Davis, R. W. and Ludford, G. S. S. (1974b) Rear stagnation-point flow in a rotating fluid. Phys. Fluids 17, 1941-1944.

DOW CORNING (1963) Information about Silicone Fluid Products from Dow Corning. Dow Corning Bulletin 05-061.

Eckhaus, W. (1973) Matched Asymptotic Expansions and Singular Perturbations. North Holland, Amsterdam, 43-96.

Greenspan, H.P. (1968) The Theory of Rotating Fluids. pp. 133-139. Cambridge University Press.

Greenspan, H. P. and Howard, L. N. (1963) On a time dependent motion of a rotating fluid. J. Fluid Mech. 17, 385-404.

Hocking, L. M. (1976) A moving fluid interface on a rough surface. J. Fluid Mech. 76, 801-817.

Hocking, L. M. (1977) A moving fluid interface. Part 2. The removal of the force singularity by a slip flow. J. Fluid Mech. 79, 209-229.

Landau, L. D. and Lifshitz, E. M. (1959) Fluid Mechanics, pp. 230-244. Pergamon Press, London.

Martínez Herranz, I. (1976) Floating zone under reduced gravity-axisymmetric equjlibrium shapes. Material Sciences in Space pp. 277-282. ESA SP 114, Paris.

Martínez Herranz, I. (1977) Floating zone-equilibrium shapes and stability criteria. Presented at $20 t h$ COSPAR Meeting, Tel-Aviv, Israel, 7-18 June 1977.

Mason, G. (1970) An experimental determination of the stable length of cylindrical liquid bubbles. $J$. Colloid Interface Sci. 32, 172-176.

Mellor, G. L., Chapple, P. J. and Stokes, V. K. (1968) On the flow between a rotating and a stationary disc. J. Fluid Mech. 31, 95-112.

Nayfeh, A. H. (1973) Perturbation Methods, pp. 159-307. Wiley, New'York.

Nigam, S. D. (1951) Rotation of an infinite plane lamina: Boundary layer growth: Motion started impulsively from rest. Quart. Appl. Math. 9, 89-91.

Padday, J. F. (1977) Private communication.

Plateau, J. A. F. (1873) Statique Experimentale et Théorique des Liquides Soumis aux Seules Forces Moléculaires, II. Gauthier-Villars, Paris.

Riddick, J. A. and Bunger, N. B. (1970) Organic Solvents, 3rd Edn. Wiley Interscience, New York.

Van Dyke, M. (1966) The circle at low Reynolds number as a test of the method of series truncation. In Applied Mechanics (Edited by Görtler, H.), pp. 1165-1169. Springer, Berlin.

Van Dyke, M. (1975) Perturbation Methods in Fiuid Mechanics, pp. 77-98. Parabolic, Stanford, California.

Watkins, W. B. and Hussey, R. G. (1973) Spin-up from rest: Limitations of the wedemeyer model. Phys. Fluids 16, 1530-1531.

Wedemeyer, E. H. (1964) The unsteady flow within a spinning cylinder. J. Fluid Mech. 20, 383-399.

Weidman, P. D. (1976) On the spin-up and spin-down of a rotating fuid. Part 1. Extending the wedemeyer model, J. Fluid Mech. 77, 685-708.

Wuest, W. (1976) Fluid dynamics of the floating zone. In Material Sciences in Space, pp. 455-465. ESA SP 114, Paris. 


\section{Appendix A}

\section{Nomenclature}

$C$ rotation parameter (Bond number), $C=\rho R^{3} \Omega^{2} / \sigma$

$E$ Ekman number, $E=\nu / \Omega R^{2}$

$L_{i}$ term in series solution for position of free surface

$P_{i}$ term in series solution for pressure in the corner region

$R$ radius of the undisturbed cylindrical floating zone

$U_{i}$ term in series solution for radial velocity in the corner region

$W_{i}$ term in series solution for normal-todisc velocity in the corner region

$f_{1}(\eta)$ first term in series solution for radial velocity in the central core

$g_{1}(\eta)$ first term in series solution for azimuthal velocity in the central core

$h_{1}(\eta)$ first term in series solution for normalto-disc velocity in the central core

1 nondimensional radial distance of the free surface to its undisturbed position

$p$ nondimensional pressure $p_{1}(n)$ first terms in series solution for nondimensional pressure in the central core

$r$ nondimensional radial distance

$t$ nondimensional time

$u$ nondimensional radial velocity

$v$ nondimensional azimuthal velocity

$w$ nondimensional normal-to-disc velocity

$z$ nondimensional distance to disc

$\Omega$ angular velocity

$\Omega_{w}$ angular velocity of the disc

$\Omega_{f}$ angular velocity of the fluid bulk

$\epsilon$ small parameter

$\eta$ nondimensional magnified distance to the disc, $\eta=z / 2 \sqrt{ }(E \epsilon \tau)$

$\nu$ liquid kinematic viscosity

$\xi$ nondimensional magnified distance to the undisturbed free surface, $\xi=$ $(r-1) / 2 \sqrt{ }(E \epsilon \tau)$

$\rho$ liquid density. Also distance to the corner of the floating zone

$\sigma$ liquid-gas surface tension coefficient

$\tau$ nondimensional magnified time, $\tau=\| / \epsilon$. 